\title{
Larval competition in Drosophila melanogaster. II. Comparing biological and competitive parameters
}

\author{
J. R. de Miranda and \\ Paul Eggleston
}

\author{
Department of Genetics, University of Liverpool, \\ P.O. Box 147, Liverpool L69 3BX, U.K.
}

\begin{abstract}
Recent developments in the analysis of density dependent competition in Drosophila melanogaster have identified two distinct parameters, namely the competitive pressure or aggression exerted by a genotype and the sensitivity or response of a genotype to such aggression. Assuming that response is more related to the efficiency of utilising available resources and aggression to the ability to acquire those resources, we attempt to relate estimates of aggression and response obtained from a range of genotypes to estimates of larval feeding rates and conversion efficiences. No significant correlations were found and we conclude that other characteristics must be involved in a more complex determination of competitive ability.
\end{abstract}

\section{INTRODUCTION}

Recent developments in the analysis of competition (Mather and Caligari, 1981, 1983; Spitters, $1983 a, 1983 b)$ make it possible to separate the effects of intra- and inter-genotypic competition in mixed cultures. The behaviour of a genotype over a density series is taken as an estimate of intragenotypic competition. By comparing the performance of a primary (indicator) genotype when mixed with an associate competitor with its performance in mono-culture, the effects of intra- and intergenotypic competition can be separated. From each pair of genotypes we can obtain two estimates of inter-genotypic competition (the effect of competitor $X$ on the performance of competitor $Y$, and the effect of $Y$ on the performance of competitor $X$ ) and from the corresponding monocultures the effects of intra-genotypic competition (Caligari, 1980). The strengths and weaknesses of competitors are relative parameters in that mixtures contain information about the competitors solely in relation to each other. Thus, two strong competitors in mixture may produce the same results as two weak competitors and all four might behave similarly in monoculture. Without further investigation of the biological basis of competitive ability, the predictive power of such experiments is reduced.

Investigations into the biological background of larval competition in Drosophila have concen- trated on the estimation of parameters of individual genotypes which can be used to predict the outcome of competition. These may then be used to define a growth equation based on the supply, acquisition and assimilation of biomass (Bakker, 1961; de Jong, 1976; Nunney, 1983). The simultaneous growth of two non-interacting strains on a limiting resource can then be modelled and the predictions compared with the observations to determine the accuracy of the model. In plant competition a variety of such models exist (e.g., Spitters and Aerts, 1983; Spitters, 1986) which include a number of other environmental parameters affecting plant growth. Another approach is to search for a biological meaning to some of the parameters estimated from competition experiments. For example, the competitive effect of an associate genotype $X$ on the density response of an indicator genotype $Y$ contains information about both $X$ and $Y$. The effect can be interpreted as the capacity of $X$ to reduce the performance of $Y$ (the aggression of $X$ ) or the sensitivity of indicator $Y$ to the presence of competitor $X$ (the response of $Y$ ). The values of aggression and response can be calculated for each genotype when placed in a variety of associations.

Previous studies by Bakker (1961) suggest that competitive ability is determined by two types of character, namely those related to resource acquisition and those to resource utilisation. The mean response of a genotype measures how much it is 
affected by the limitations of the environment and might therefore be related to those characteristics associated with the utilisation of resources after acquisition. Mean aggression, measures the ability of a genotype to impose such limitations, and might therefore be more related to the acquisition of resources.

Here we report the competitive characteristics of genotypes previously investigated with respect to a variety of biological parameters which might be related to competition (de Miranda and Eggleston, 1987b). Correlation analyses are used to define the relationships between the biological parameters (larval feeding rate and larva to adult conversion efficiency) and the competitive parameters (aggression and response).

\section{EXPERIMENTAL DETAILS}

The experimental procedures used are identical to those of de Miranda and Eggleston (1987a). In short, competition took place in glass vials each with $5.0 \mathrm{ml} 2$ per cent bacto agar as a non nutritive base, and $55.0 \mathrm{mg}$ live yeast (Sigma YSC-2) as the food source. The wild type inbred strains used in the experimental conditions are identical to those used for the determination of the larval feeding parameters (de Miranda and Eggleston, 1987b).

These eight strains are a subset of two separate sets of substitution lines and have the following chromosome composition:

\begin{tabular}{lllllllll}
\multicolumn{1}{c}{$\begin{array}{l}\text { Chromosome } \\
\text { type }\end{array}$} & 27A & 27B & 27C & 27D & 25A & 25B & 25C & 25D \\
\hline $\mathrm{X}$ & T27 & T27 & T27 & T27 & T25 & T25 & T25 & T25 \\
II & T5 & T5 & T27 & T27 & T19 & T19 & T25 & T25 \\
III & T5 & T27 & T5 & T27 & T19 & T25 & T19 & T25
\end{tabular}

Where T5, T27, T19 and T25 refer to four inbred lines derived from the Texas population (Linney et al., 1971).

Two yellow marked strains $\left(y^{2} \mathrm{~T} 19\right.$ and $\left.y^{2} \mathrm{~T} 25\right)$ were used as testers for the wild type strains in the mixed cultures and the yellow body colour is used to separate the competitors in duoculture. Previous studies have shown that the $y^{2}$ allele itself has little effect on the competitive behaviour of the strains (Caligari, 1980; Mather and Caligari, 1981; Eggleston, 1987).

For each of the genotypes one monoculture density series (30, 60, 90 and 120 eggs/culture) and all possible duoculture combinations of wild type and yellow strain were raised following the replacement density series of de Wit (1960), with $(30: 90),(60: 60)$ and $(90: 30)$ wild type:yellow eggs/culture. All cultures in each experiment were individually randomised and incubated at $25^{\circ} \mathrm{C} \pm$ $0.5^{\circ} \mathrm{C}$. The experiment was repeated four times to provide a source of error variation. The adults were collected daily for 10 days from each culture, and were separated by body colour, counted and weighed.

Two competitive parameters were investigated.

$p=$ proportion of seeded eggs of a genotype surviving to adulthood.

$\bar{w}=$ mean weight of the surviving adults of each genotype.

$p$ was transformed to $p_{a}$ using the angular transformation (Bartlett 1947) and $\bar{w}$ was transformed to $1 / \bar{w}$ for regression purposes (de Miranda and Eggleston, 1987a).

The results were analysed using the multiple linear regression procedure of Mather and Caligari (1981). Fig. 1 defines the parameters obtained from

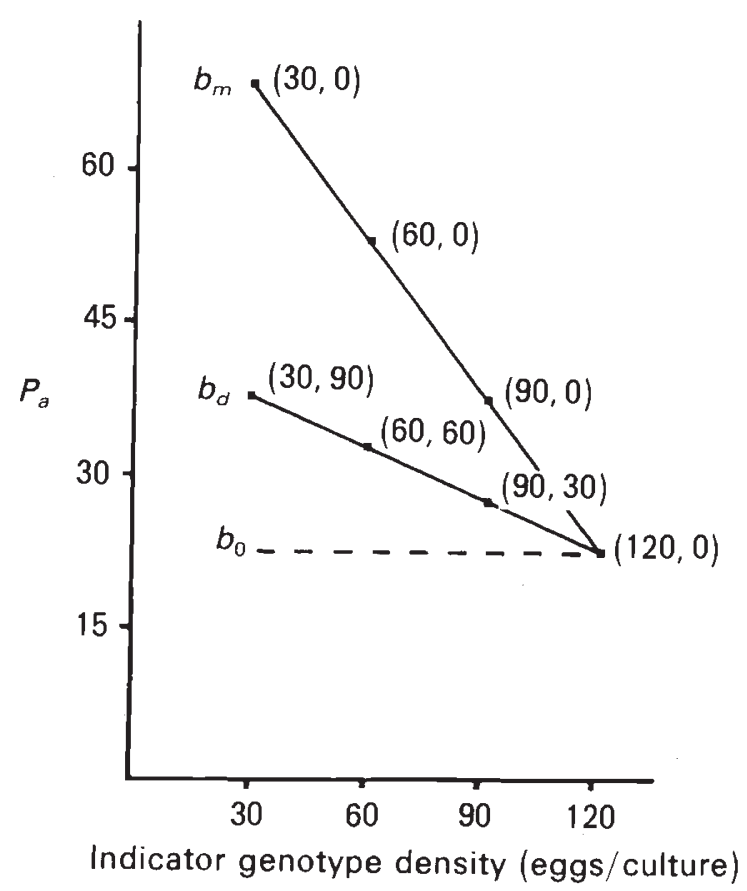

Figure 1 Graphic representation of the regression parameters. $b_{m}$ and $b_{d}$ refer to the slopes obtained in monoculture and duocultures respectively. $b_{0}$ is the line of zero relationship from which $b_{m}$ can be measured and $e$ refers to the expression of the character at the reference density $(N)$ of 120 eggs per culture. The figures in brackets at each plot represent the number of indicator and associate eggs per culture respectively, and the total number of eggs in the duocultures is always equal to $N$ (120 eggs per culture). 
such an analysis. The slope $b_{m}$ is the response of a genotype to density in monoculture and the slope $b_{d}$ is its response in duoculture. The frame of reference is completed by the horizontal line of zero relationship $\left(b_{0}\right)$. Intra-genotypic competition is estimated by $\left(b_{0}-b_{m}\right)$, while the intergenotypic effect of the associate genotype on the indicator genotype is estimated by $\left(b_{d}-b_{m}\right)$. These estimates are referred to as $c$-values. The parameter $e$ is an estimate of the expression of the character at the reference density of 120 eggs/culture (de Miranda and Eggleston, 1987a).

\section{RESULTS}

There is considerable variation between the genotypes in their competitive behaviour for both $p_{a}$ and $1 / \bar{w}$, as shown by the $c$-values in table 1 . The values along the leading diagonal measure the intra-genotypic competitive ability of each genotype and the $c$-values in the bottom two rows refer to the competitive effects of the wild type strains as associate competitors, measuring the aggression of these genotypes against each of the yellow marked tester strains. The $c$-values in the right hand columns refer to the competitive response of the wild type strains to inter-genotypic competition from the two yellow marked testers. Estimates of the mean aggression and mean response of the eight wild type strains, obtained by pooling the items in each column and each row respectively, are given in table 2. As previously found (Mather and Caligari, 1983, Eggleston, 1985, de Miranda and Eggleston, 1987a) there is considerable variation between the genotypes for mean aggression and mean response $\left(\chi_{(7)}^{2}=170 \cdot 3\right.$ and $\chi_{(7)}^{2}=374.7$ respectively for the character $p_{a}$; $\chi_{(7)}^{2}=246.7$ and $\chi_{(7)}^{2}=64 \cdot 1$ respectively for the character $1 / \bar{w}$, all of which are highly significant). These earlier studies also indicated that mean aggression and mean response varied independently. However, in this study the correlations between mean aggression and mean response were $r_{6}=0.93$ for $p_{a}$ and $r_{6}=0.78$ for $1 / \bar{w}(P<0.001$ and $P<0.05$ respectively). As a third of each estimate of mean aggression arises from the value for intra-genotypic competition and this value is also included in the estimate of mean response a positive correlation was not unexpected. Exclusion of the values for intra-genotypic competition from estimates of mean aggression and mean response reduces the correlation coefficients to $r_{6}=0.62$ and $r_{6}=0.11$ for $p_{a}$ and $1 / \bar{w}$ respectively, neither of which is significant.
Table 3 shows the estimates and variances of the $e$-values of the eight wild type genotypes for $p_{a}$ and $1 / \bar{w}$. Significant genotypic variation exists for both $p_{a}$ and $1 / \bar{w}\left(\chi_{(7)}^{2}=84.916\right.$ and $\chi_{(7)}^{2}=$ $523 \cdot 161$ respectively). The relationships between the biological parameters defined in the accompanying paper (de Miranda and Eggleston, 1987b) and the estimates of aggression and response are described in table 4. The estimates of aggression and response used are those that exclude the values for intra-genotypic competition since this improves both the primary and partial correlations. Only larval feeding rates $\left(b_{53}\right.$ and $\left.b_{77}\right)$ and larva-adult conversion efficiencies $\left(n_{53}\right.$ and $\left.n_{77}\right)$ are considered in table 4. Larval conversion efficiency (also investigated by de Miranda and Eggleston, 1987 b) showed little correlation with either competitive parameter, and was excluded from further analysis.

Table 4 documents a set of partial correlations, corrected for both the correlation between aggression and response and for the correlation between the two biological parameters. The partical correlations therefore give an unbiased description of the relationship between the biological parameters and our estimates of aggression and response. If these estimates of aggression and response were, in fact, statistical measures of the acquisition and utilisation characters defined by Bakker (1961) we might expect response to be negatively correlated to feeding rate and conversion efficiency since a high response would indicate sensitivity to competition and hence inefficiency. We might expect aggression, however, to be positively correlated to the biological parameters since it is a measure of competitive strength. It is clear from table 4 that no such consistent trends are evident and indeed, the majority of the coefficients are not significant. We conclude that there is no simple relationship between the competitive parameters (aggression and response) and the biological parameters (feeding rate and conversion efficiency).

\section{DISCUSSION}

Any comparison between genotypes in competitive ability assumes that all larval mortality is due to the effects of competition and that at low densities survival is high and near the percentage egg hatchability. This however, is not the case. Genotypes $25 \mathrm{~A}$ and $25 \mathrm{C}$ in particular show high levels of non-competitive death, resulting in a low $e$ value and a near absence of intra-genotypic competition for $p_{a}$, and a similar, though not so 


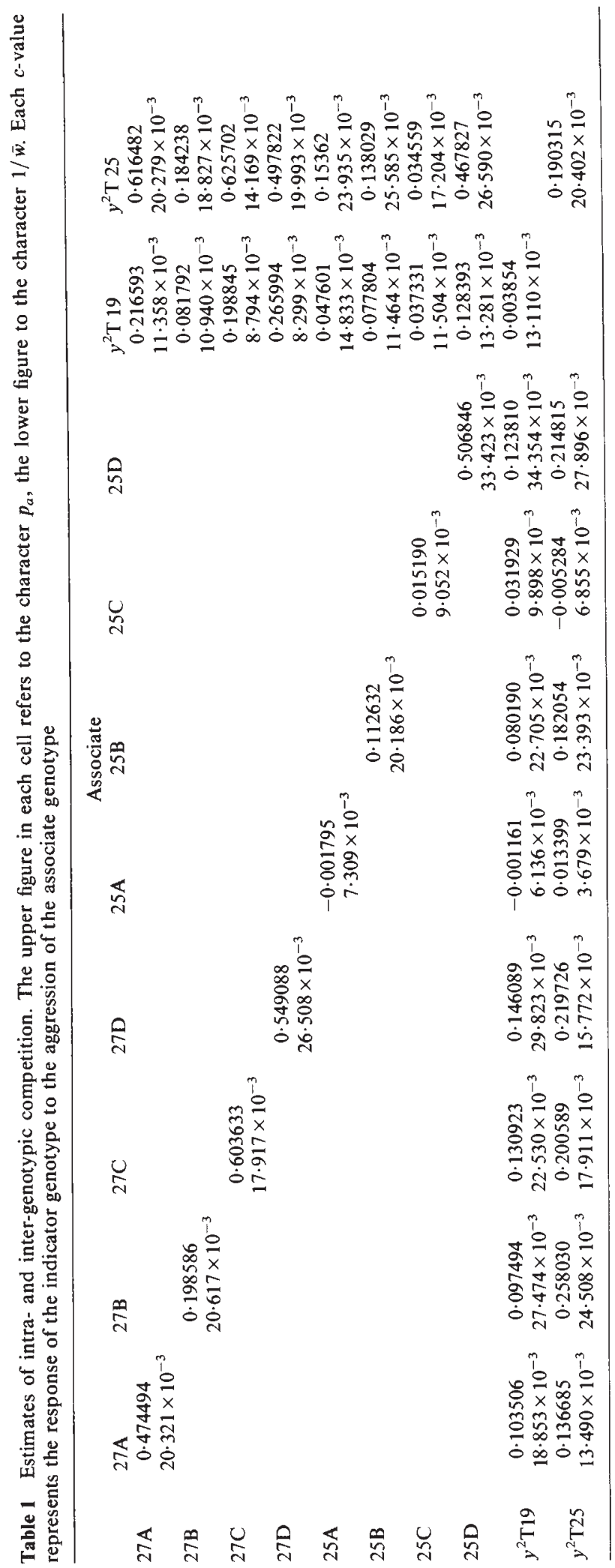


Table 2 Estimates and variances for mean aggression and mean response values obtained for the eight genotypes in this experiment. The upper and lower entries in each cell refer to the characters $p_{a}$ and $1 / \bar{w}$ respectively

\begin{tabular}{llclc}
\hline & $\begin{array}{l}\text { Mean } \\
\text { aggression }\end{array}$ & var $\times 10^{-5}$ & $\begin{array}{l}\text { Mean } \\
\text { response }\end{array}$ & var $\times 10^{-5}$ \\
\hline $27 \mathrm{~A}$ & 0.23823 & 91.2092 & 0.4385 & $120 \cdot 2839$ \\
& 0.01756 & 0.30807 & 0.07732 & 0.40585 \\
$27 \mathrm{~B}$ & 0.18470 & 76.0377 & 0.15487 & 57.9736 \\
& 0.02419 & 0.24246 & 0.01680 & 0.13085 \\
$27 \mathrm{C}$ & 0.31172 & 70.4626 & 0.47610 & $67 \cdot 7589$ \\
& 0.01945 & 0.22683 & 0.01363 & 0.12001 \\
$27 \mathrm{D}$ & 0.30497 & 84.6741 & 0.43763 & 92.8241 \\
& 0.02403 & 0.21926 & 0.01827 & 0.03722 \\
25A & 0.00348 & 37.6399 & 0.06739 & 22.7513 \\
& 0.00571 & 0.16646 & 0.01536 & 0.11417 \\
$25 \mathrm{~B}$ & 0.12496 & 40.4495 & 0.10949 & 34.0893 \\
& 0.02210 & 0.16669 & 0.01908 & 0.11509 \\
$25 \mathrm{C}$ & 0.01395 & 49.0754 & 0.02903 & 68.8079 \\
& 0.00860 & 0.10328 & 0.01259 & 0.10133 \\
$25 \mathrm{D}$ & 0.27590 & 46.4550 & 0.36769 & 58.3229 \\
& 0.03189 & 0.17686 & 0.02443 & 0.15615 \\
\hline
\end{tabular}

extreme case for $1 / \bar{w}$. Since the linear component of the critical curve for the mean adult weight $(\bar{w})$ extends over a large range of densities (Bakker, 1961) the effect of intra-genotypic competition on $\bar{w}$ may be unbiased although its $e$ value may be displaced. For $p_{a}$ the critical curve is contained within a far narrower range of densities (Bakker, 1961; Kearsey, 1967) and the presence of noncompetitive larval mortality may have serious repercussion on the estimation of the effects of intra-genotypic competition. Both de Jong and Nunney's models for larval growth (de Jong, 1976; Nunney, 1983) allow for larval mortality independent of competitive mortality, but do not relate this to any particular instar. Clearly, if noncompetitive larval mortality occurred near the end of the larval phase, after food exhaustion, the effect of this on the density response in $p_{a}$ would merely be to shift the $e$-value. Early larval death would reduce the effective density and increase the chances of survival for the remaining larvae. The low $e$ value for $p_{a}$ and a low $e$ value for $1 / \bar{w}$ (indicating a large average adult weight at the highest density) suggest that this is indeed true for genotypes $25 \mathrm{~A}$ and $25 \mathrm{C}$. Larval mortality may have a more serious effect on the estimation of intergenotypic competition. Early death of a significant proportion of one genotype means that the extra food available is shared between the two groups of competitors according to their abilities to acquire the resource, which artificially enhances the prospects of survival and may produce a high adult weight for both. Much depends on the actual timing of larval mortality, the competitive relation-

Table 3 Estimates of $(e)$ the mean character expression at the reference density of 120 eggs pler culture for characters $p_{a}$ and $1 / \bar{w}$, together with their variances

\begin{tabular}{lllll}
\hline & & $p_{a}$ & & \multicolumn{2}{c}{$1 / \bar{w}$} \\
& $e$ & $\operatorname{var}(e)$ & $e$ & $\operatorname{var}(e)$ \\
\hline $27 \mathrm{~A}$ & 31.3550 & 9.74305 & 3.47559 & 0.32874 \\
$27 \mathrm{~B}$ & 43.6406 & 4.69587 & 3.24044 & 0.010599 \\
$27 \mathrm{C}$ & 25.2323 & 5.48847 & 3.12308 & 0.009721 \\
$27 \mathrm{D}$ & 29.2647 & 7.51877 & 4.17846 & 0.030144 \\
$25 \mathrm{~A}$ & 26.9655 & 1.84827 & 1.83333 & 0.009248 \\
$25 \mathrm{~B}$ & 46.6855 & 2.76123 & 3.20895 & 0.009323 \\
$25 \mathrm{C}$ & 28.6167 & 5.58065 & 1.77694 & 0.008208 \\
$25 \mathrm{D}$ & 28.7558 & 4.72415 & 4.96044 & 0.012648 \\
\hline
\end{tabular}


Table 4 Partial correlation coefficients for $4 \mathrm{df}$ each between the competitive parameters (rows) and the biological parameters (columns). The top two rows refer to the mean aggression $(A)$ and mean response $(R)$ for $p_{a}$, while the lower two rows refer to the mean aggression and response for $1 / \bar{w}$. All of these estimates exclude the values for intra-genotypic competition. $b_{53}$ and $b_{77}$ refer to the 2nd and 3 rd instar feeding rates while $n_{53}$ and $n_{77}$ refer to the 2nd and 3 rd instar larva-adult conversion efficiency. The partial correlations were corrected for both the correlation between aggression and response for $p_{a}$ and $1 / \bar{w}$ separately, and for the correlation between the feeding rate and the conversion efficiency at each instar stage

\begin{tabular}{llllr}
\hline & $b_{53}$ & \multicolumn{1}{c}{$b_{77}$} & $n_{53}$ & \multicolumn{1}{c}{$n_{77}$} \\
\hline$A$ & 0.278 & 0.177 & 0.063 & 0.165 \\
$R$ & 0.005 & -0.304 & 0.646 & 0.131 \\
$A$ & 0.616 & -0.150 & 0.328 & 0.165 \\
$R$ & 0.775 & $0.841^{*}$ & -0.212 & -0.503 \\
\hline
\end{tabular}

* The significance levels are given as $*=P<0.05$.

ship at that time, and the influence of extra food on the length of the instar stages for each genotype.

The basic assumption of these experiments was that competitive ability is largely determined by a few major biological parameters, operating during the larval stage. Most of the available food is probably consumed by second and early third instars making these two stages the main targets for a study of the biological parameters. Correlation of the food acquisition and utilisation capabilities of these stages with the competitive parameters of aggression and response might then provide a quick means of testing individual genotypes for their relative competitive abilities. However, the lack of a significant correlation between aggression and larval feeding rates, and between adult or larval conversion efficiences and response suggest that the determination of competitive ability is not as simple as this. For the character $p_{a}$ the absence of any meaningful correlation can be ascribed to the nature of the character. The central biological characteristic necessary for survival is the attainment of a minimum larval weight required for successful pupation, which varies between genotypes (Bakker, 1961). For the character $1 / \bar{w}$ attainment of the minimum pupation weight is less critical in determining the relative competitive abilities, since the character is not so much concerned with survival, as with the larval growth after survival has been assured. Possibly as a result, the relevant correlation coefficients between the biological and the competitive parameters are higher for this character than for $p_{a}$.

Although the biological parameters estimated are undoubtedly important in determining the overall competitive phenotype, they do not override other aspects of larval growth. It would, therefore, be futile to postulate a general larval growth model based on them since too many factors remain unknown. These include the duration of the various instar stages, with the levels of density independent mortality in each stage, (Sokal and Bryant, 1967) the minimum larval weights required for pupation and the larval feeding rates in the late third instar. In addition larval growth may be influenced by random variability in the timing of the larval moults which might produce the large and heterogenous replication errors associated with the third instar feeding rates. Altapov's early study of the larval instar stages (Altapov, 1929) shows a large difference between replicates in the mean transitional time between second and third instar larvae, without change in the spread around these means, suggesting that whatever caused the shift has affected all larvae similarly. If this similarity extends to different genotypes we would find a correlated growth pattern in duocultures that would make nonsense of individual parameters, estimated at defined time intervals, whilst leaving the relative competitive abilities of the competitors unimpaired. Robertson's ecological study of the relationships between the length of the larval period and adult body weight on sub-optimal diets (Robertson, 1960a, 1960b, 1960c, 1963, 1964) concludes that both individual adaptation and genotype selection in a limiting environment can combine in many different ways to maximise survival. The competitive ability of individuals and genotypes may have a similarly diverse and flexible biological basis.

\section{REFERENCES}

ALTAPOV, w. W., 1929. Growth and variation of the larvae of Drosophila melanogaster. J. Expt. Zool., 52, 407-437.

BAKKER, K. 1961. An analysis of factors which determine success in competition for food among larvae of Drosophila melangoaster. Arch. Neerl. Zool., 14, 200-281.

BARTLETT, M. S., 1947. The use of transformations. Biometrics, $3,39-53$.

CALIGARI, P. D. S. 1980. Competitive interactions in Drosophila melanogaster. I. Monocultures. Heredity, 45, 219-231.

EGGLESTON, P. 1985. Variation for aggression and response in the competitive interactions of Drosophila melanogaster. Heredity, 54, 43-51.

EGGLESTON, P. 1987. The use of genetic markers in the analysis of competitive interactions in Drosophila melanogaster. Genetica (in press).

DE JONG, G. 1976. A model of competition for food. I. Frequency dependent selection. Am. Nat., 110, 1013-1027.

KEARSEY, M. J. 1965. The interaction of competition and the supply of food in two lines of Drosophila melanogaster. Heredity, 20, 169-181. 
LINNEY, R., BARNES, B. W. AND KEARSEY, M. J. 1971. Variation for metrical characters in Drosophila populations. Heredity, 46, 239-254.

MATHER, K. AND Caligari, P. D. S. 1983. Pressure and response in competitive interactions. Heredity, 54, 435-454.

NUNNEY, L. 1983. Sex differences in larval competition in Drosophila melanogaster. The testing of a competition model and its relevance to frequency dependent selection. Am. Nat., 121, 67-93.

DE MIRANDA, J. R. AND EGGLESTON, P. 1987a. A comparison of substitution and addition designs for the analysis of competitive interactions in Drosophila melanogaster. Heredity, 58, 279-288.

DE MIRANDA, J. R. AND EGGLESTON, P. 1987b. Larval competition in Drosophila melanogaster. I. Estimation of larval growth parameters. Heredity, 60, 205-212.

ROBERTSON, F. W. $1960 a$. The ecological genetics of growth in Drosophila. 1. Body size and developmental time on different diets. Gen. Res. Camb., 1, 288-304.

ROBERTSON, F. W. $1960 \mathrm{~b}$. The ecological genetics of growth in Drosophila. 2. Selection for large body size on different diets. Gen. Res. Camb., 1, 305-318.

ROBERTSON, F. W. $1960 c$. The ecological genetics of growth in Drosophila. 3. Growth and competitive ability of strains selected on different diets. Gen. Res. Camb., 1, 333-350.
ROBERTSON, F. W. 1961. The ecological genetics of growth in Drosophila. 4. Influence of larval nutrition on the manifestation of dominance. Gen. Res. Camb., 2, 346-360.

ROBERTSON, F. W. 1963. The ecological genetics of growth in Drosophila. 6. The genetic correlation between the duration of the larval period and body size in relation to larval diet. Gen. Res., Camb., 4, 74-92.

ROBERTSON, F. W. 1964. The ecological genetics of growth in Drosophila. 7. The role of canalisation in the stability of growth relations. Gen. Res. Camb., 5, 107-126.

SPITTERS, C. J. T. $1983 a$. An alternative approach to the analysis of mixed cropping experiments. 1. The estimation of competition effects. Neth. J. Agric. Sci., 31, 1-11.

SPITTERS, C. J. T. $1983 \mathrm{~b}$. An alternative approach to the analysis of mixed cropping experiments. 2. Marketable yield. Neth. J. Agric. Sci., 31, 143-155.

SPITTERS, C. J. T. AND AERTS, R. 1983. Simulation of competition for light and water in crop-weed associations. Asp. Appl. Biol., 4, 467-483.

SPITTERS, C. J. T. 1986. A confrontation between a descriptive and an explanatory approach to inter-genotypic competition. Proc. VIth meeting Eucarpia section Biometrics in Plant Breeding, 205-277.

DE WIT, C. T. 1960. On competition. Versl. Landb. Onderz., 66, $1-82$. 